\title{
Synthesis and Properties of Fumed Silicas Modified with Mixtures of Poly(methylphenylsiloxane) and Dimethyl Carbonate
}

\author{
Iryna S. Protsak ${ }^{1}$, Valentyn A. Tertykh${ }^{1}$, Yulia M. Bolbukh ${ }^{1}$, Dariusz Sternik ${ }^{2}$, \\ Anna Derylo-Marczewska² \\ ${ }^{1}$ Department of Chemisorption, Chuiko Institute of Surface Chemistry of National Academy of Sciences of \\ Ukraine, Kiev, Ukraine \\ ${ }^{2}$ Faculty of Chemistry, Maria Curie-Sklodowska University, Lublin, Poland \\ Email: "iryna protsak@yahoo.com
}

Received 16 November 2015; accepted 18 December 2015; published 21 December 2015

Copyright (C) 2015 by authors and Scientific Research Publishing Inc.

This work is licensed under the Creative Commons Attribution International License (CC BY).

http://creativecommons.org/licenses/by/4.0/

(c) $\stackrel{\text { (i) }}{\mathrm{BY}}$ Open Access

\begin{abstract}
Effect of the concentration ratios of organosiloxane/initiator and treatment temperature on the characteristics of hydrophobic products obtained by modification of surface of fumed silica with poly(methylphenylsiloxane) (PMPS) in the presence of dimethyl carbonate has been studied. Morphology, particle size, surface area and coating microstructure of modified silicas were analyzed by methods of transmission electron and atomic force microscopies, nitrogen adsorption-desorption data. Carbon contents in the grafted modifying layer of organosilicas were determined using IR spectroscopy and elemental analysis. Hydrophilic-hydrophobic properties of surface of the obtained modified silicas were estimated by measurements of contact angles of wetting. It was shown that modification of pyrogenic silicas with mixtures of poly(methylphenylsiloxane) and dimethyl carbonate allows to obtain the homogeneous hydrophobic products and serve their nanodispersity.
\end{abstract}

\section{Keywords}

Fumed Silica, Poly(methylphenylsiloxane), Dimethyl Carbonate, Surface Modification, Hydrophilic-Hydrophobic Properties

\section{Introduction}

Modified disperse silicas with grafted phenyl groups are applied as the stationary phases and carriers in chro-

${ }^{*}$ Corresponding author.

How to cite this paper: Protsak, I.S., Tertykh, V.A., Bolbukh, Y.M., Sternik, D. and Derylo-Marczewska, A. (2015) Synthesis and Properties of Fumed Silicas Modified with Mixtures of Poly(methylphenylsiloxane) and Dimethyl Carbonate. World Journal of Nano Science and Engineering, 5, 152-160. http://dx.doi.org/10.4236/wjnse.2015.54017 
matography, and as the fillers for manufacturing adhesives, coatings and materials with high heat resistance [1]-[3]. Appropriate phenyl-containing alkoxy and chlorosilanes are mainly used for preparation of such type of modified silicas. However, the case of phenyl-containing alkoxysilanes chemisorption of alcohol molecules is observed as a side process at high temperatures of silica treatment. Using the phenyl-containing chlorosilanes for surface modification is not always suitable because of the elimination of hydrogen chloride from reaction with silanol groups of silica. It may result in the presence of potential electrolytes in the obtained modified products. Therefore, application of the phenyl-containing siloxane oligomers for modification of silica surface is undoubtedly interesting. However, the processes of chemisorption involving silanol groups of the silica surface and oligomeric phenylsiloxanes proceed at high temperatures $\left(>350^{\circ} \mathrm{C}\right)$.

It is known from the experiment [4] [5] that due to interaction between dimethyl carbonate and polydimethylsiloxane, a reaction product is formed with viscosity considerably lesser than those of parent substances, so a conclusion can be made on the rupture of chemical bonds in polydimethylsiloxane chains, and it is established [6] that in the presence of dimethyl carbonate, chemisorption of poly(dimethylsiloxane) on the silica surface is carried out at lower temperatures.

Dimethyl and diethyl carbonates as it was earlier established are effective reagents in the reaction of siloxane bond cleavage in poly(dimethylsiloxanes) [4] [7] [8]. Thus, it can be expected that the simultaneous use of dimethyl carbonate, which belongs to "green chemistry" solvents and reagents [9] [10], and phenyl-containing polysiloxane in the processes of chemical modification of silica will provide deoligomerization of siloxane and promote to cleavage of siloxane bridges on the silica surface.

In this work, such approach was tested in the simultaneous application of dimethyl carbonate and poly(methylphenylsiloxane) (PMPS) for modification of the silica surface. Special attention was paid for investigation of structural and surface characteristics of the products obtained during modification of fumed silica with the mixtures of different composition.

\section{Experimental}

\subsection{Reagents}

Fumed silica characterized by a specific surface area of $260 \mathrm{~m}^{2} / \mathrm{g}$ (A-300, Kalush, Ukraine), synthesized via high-temperature hydrolysis of silicon tetrachloride was taken for surface modification. As a modifying reagent poly(methylphenylsiloxane) fluid PMPS-4 (Zaporizhzhya, Ukraine, GOST 15866-70) with degree of polymerization $n=8-10$ was used. The applied organosilicon polymer is characterized by a wide temperature (from $-20^{\circ} \mathrm{C}$ to $+350^{\circ} \mathrm{C}$ ) range of stability, chemical inertness and hydrophobic properties. Dimethyl carbonate (DMC) was supplied by Sigma Aldrich.

\section{Synthesis of Composite Materials}

Modification of fumed silica surface with PMPS was performed at the different temperatures for 2 hrs with or without the addition of dimethyl carbonate (DMC). The modification process was performed in a glass reactor with a stirrer at rotational speed from 20 to $300 \mathrm{ppm}$. After loading with fumed silica, all air volume in the reactor was filled with nitrogen and the reactor was heated up to a defined temperature. Then, nitrogen supply was interrupted and the modifying reagent was added into reactor by means of its aerosol spraying through a nozzle.

Four series of the samples were synthesized. $10 \mathrm{~g}$ of fumed silica were treated with $2.2 \mathrm{~g}$ of PMPS in the first series. In these second series the surface treatment was carried out with the mixture of PMPS and DMC at the weight ratios $\mathrm{SiO}_{2}: \mathrm{PMPS}: \mathrm{DMC}=10: 2.2: 1.5$. The weight ratios $\mathrm{SiO}_{2}: \mathrm{PMPS}: \mathrm{DMC}=10: 2.2: 1.0$ were used in the third series. Modification of the surface was carried out with the mixture of the PMPS and DMC in the weight ratios $\mathrm{SiO}_{2}$ :PMPS:DMC = 10:2.2:0.5 in the fourth series. Modification of the fumed silica surface was performed at three different temperatures: $200^{\circ} \mathrm{C}, 250^{\circ} \mathrm{C}$ and $300^{\circ} \mathrm{C}$. Removal of the physically adsorbed reactants was carried out in a Soxhlet apparatus with $n$-hexane as a solvent at $68^{\circ} \mathrm{C}$ for $1 \mathrm{~h}$. Then, the washed sample was dried at $80^{\circ} \mathrm{C}$ for $2 \mathrm{hrs}$.

\subsection{Methods}

\subsubsection{Infrared Spectra}

In order to control the flow of surface reactions, IR spectra were recorded using a Specord M-80 spectrophoto- 
meter in a range of wave numbers $4000-200 \mathrm{~cm}^{-1}$. The silica samples were pressed into rectangular $28 \times 8 \mathrm{~mm}$ plates of $25 \mathrm{mg}$ weight.

\subsubsection{Nanostructural Characterization}

Surface microstructure and morphology as well as coating homogeneity for the modified samples were analyzed by transmission electron microscopy, TEM (Tecnai G2T20 X-TWIN, USA) and atomic force microscopy, AFM (Nanoscope V Digital Instruments, USA, with a Tapping Mode technique). AFM data processing was performed using the SPIP program (version 5.0.6).

\subsubsection{Nitrogen Adsorption Measurements}

Porous structure (specific surface area, pore volume and pore size distribution function) was characterized using nitrogen adsorption-desorption data measured using Accelerated Surface Area and Porosimetry analyzers ASAP 2020 and 2420 (Micromeritics, USA). Before the measurements, the samples were outgassed at $110^{\circ} \mathrm{C}$. The specific surface area, $S_{\mathrm{BET}}$, was calculated using the BET method [11]. The total pore volume $V_{p}$ was evaluated using the nitrogen adsorption data at the relative pressure $p / p_{0} \sim 0.98-0.99$, where $p$ and $p_{\mathrm{o}}$ are equilibrium pressure and vapor pressure at the temperature $77.4 \mathrm{~K}$, respectively. Pore size distribution (PSD) was calculated by employing the regularization approach at the fixed regularization parameter $\alpha=0.01$ according to the Nguyen-Do method for the bimodal PSD [12]-[14]. This method was used previously to describe the structural properties of carbonaceous materials [15]-[18], composites [14] [19] [20] and aluminosilicates [21]. In the paper the model of the pores as voids between spherical particles and the model of the cylindrical pores were used.

\subsubsection{Contact Angle Measurements}

Hydrophilic-hydrophobic properties of the surface of obtained modified silicas were estimated by measurements of contact angles of water drops. The contact angle data were measured using a commercial Contact Angle Meter (GBX Scientific Instruments, France) equipped with a temperature and humidity controlled measuring chamber and a digital camera $\left(\mathrm{T}=20^{\circ} \mathrm{C}\right.$; $\left.\mathrm{RH}=50 \%\right)$.

\subsubsection{Elemental Analysis}

To measure the content of grafted organic groups in the synthesized samples, the Perkin-Elmer $2400 \mathrm{CHN}$ analyzer (USA) was used. The modifying layer was oxidized to produce $\mathrm{H}_{2} \mathrm{O}$ and $\mathrm{CO}_{2}$ during the samples heating in the oxygen flow at $750^{\circ} \mathrm{C}$.

\section{Results and Discussion}

Control of the surface reactions under the surface modification of the fumed silica with poly(methylphenylsiloxane) and dimethyl carbonate was performed by IR spectroscopy. IR spectra of the silicas modified with PMPS are shown in Figure 1. Surface treatment was performed at $200^{\circ} \mathrm{C}, 250^{\circ} \mathrm{C}$ and $300^{\circ} \mathrm{C}$ for $2 \mathrm{hrs}$. High intensity bands at $2900-3100 \mathrm{~cm}^{-1}$ (asymmetric stretching $\mathrm{C}-\mathrm{H}$ vibrations in methyl and phenyl groups) and accompanying band at $2910 \mathrm{~cm}^{-1}$ (symmetric stretching $\mathrm{C}-\mathrm{H}$ vibrations) are observed in the IR spectra of the samples of fumed silica modified with poly(methylphenylsyloxane) at $200^{\circ} \mathrm{C}, 250^{\circ} \mathrm{C}$ and $300^{\circ} \mathrm{C}$ (spectra 3 - 5). The samples of silicas modified at $200^{\circ} \mathrm{C}$ and $250^{\circ} \mathrm{C}$ (spectra 3, 4) are characterized by the highest content of methyl groups and it is correlated with data of elemental analysis (Table 1).

IR spectra are also characterized by the presence of a broad absorption band at $3600-3000 \mathrm{~cm}^{-1}$ corresponding to $\mathrm{O}-\mathrm{H}$ vibrations in adsorbed water molecules and in silanol groups which formed hydrogen bonds with molecules of adsorbate. The band at $3750 \mathrm{~cm}^{-1}$ corresponding to $\mathrm{O}-\mathrm{H}$ stretching vibrations of the free silanol groups [22] [23] is intense in the spectrum of the original silica, but this band is not seen in the spectra of the modified silicas. The band of deformation vibrations of the adsorbed water (at $1630 \mathrm{~cm}^{-1}$ ) is observed in all three spectra of the modified samples (spectra 3-5), but this band is the most intensive in the spectrum of the pristine silica (spectrum 1).

Carrying out the process of modification in the simultaneous presence of PMPS and dimethyl carbonate in the reaction mixture results in both the increase of intensity of the bands of stretching vibrations of $\mathrm{C}-\mathrm{H}$ bonds in the methyl groups in the wave number range $3000-2900 \mathrm{~cm}^{-1}$ [24] and the increase of intensity of the bands of stretching vibrations of $\mathrm{C}-\mathrm{H}$ bonds in the phenyl groups manifested in the frequency range of $3100-3000 \mathrm{~cm}^{-1}$ 


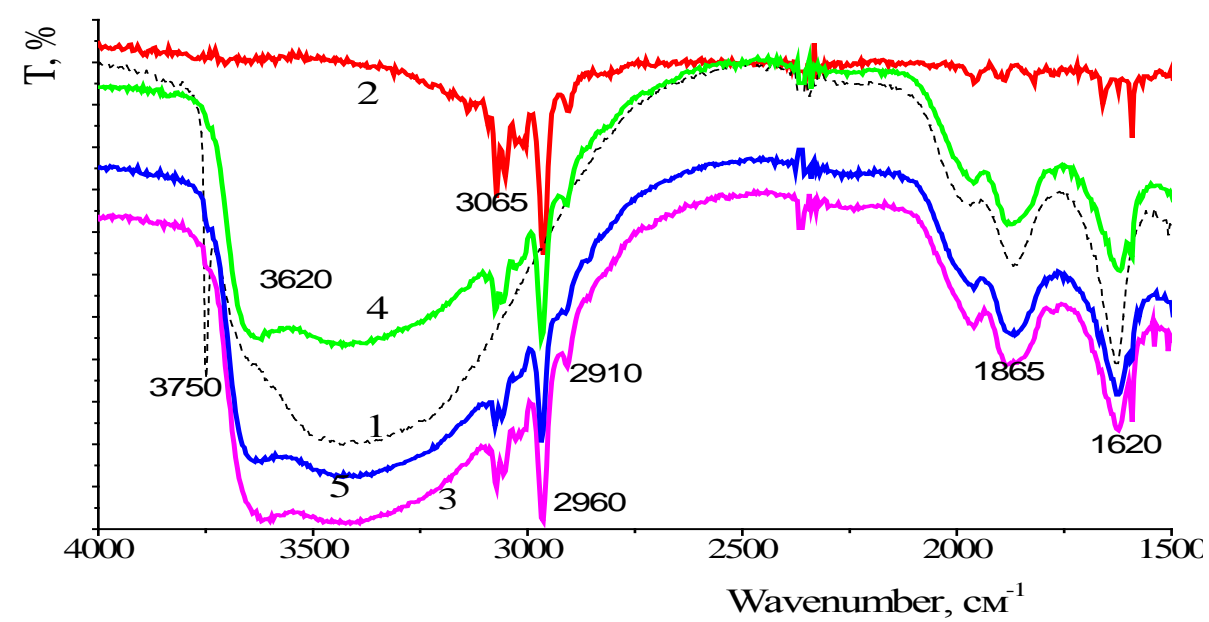

Figure 1. IR spectra of the original fumed silica A-300 (1), PMPS (2), and the fumed silicas modified with PMPS for 2 hrs at $200^{\circ} \mathrm{C}, 250^{\circ} \mathrm{C}$ and $300^{\circ} \mathrm{C}$ (3 - 5, respectively).

Table 1. Structural characteristics of fumed silicas modified with PMPS and its mixtures with DMC determined from nitrogen adsorption, and hydrophilic-hydrophobic properties of the surface determined on the basis of elemental analysis and values of contact angles of wetting.

\begin{tabular}{|c|c|c|c|c|c|c|}
\hline $\mathrm{T},{ }^{\circ} \mathrm{C}$ & Component ratios & $\mathrm{S}_{\mathrm{BET}}, \mathrm{m}^{2} / \mathrm{g}$ & $\mathrm{V}_{\mathrm{p}}, \mathrm{cm}^{3} / \mathrm{g}$ & $\mathrm{R}_{\text {ave }} \mathrm{nm}$ & Carbon content, wt.\% & $\begin{array}{c}\text { Contact angle of } \\
\text { wetting } \Theta \text {, degrees }\end{array}$ \\
\hline \multirow[b]{4}{*}{200} & Pristine $\mathrm{SiO}_{2}$ & 260 & 0.539 & 11 & 0 & 0 \\
\hline & $\mathrm{SiO}_{2}+\mathrm{PMPS}(10 \mathrm{~g}+2 \mathrm{ml})$ & 102 & 0.024 & 40 & 7.3 & 133 \\
\hline & $\mathrm{SiO}_{2}+\mathrm{PMPS}+0.5 \mathrm{ml} \mathrm{DMC}$ & 151 & 0.021 & 15 & 6.0 & 130 \\
\hline & $\mathrm{SiO}_{2}+\mathrm{PMPS}+1.0 \mathrm{ml} \mathrm{DMC}$ & 158 & 0.021 & 15 & 6.0 & 130 \\
\hline \multirow{4}{*}{250} & $\mathrm{SiO}_{2}+\mathrm{PMPS}+1.5 \mathrm{ml} \mathrm{DMC}$ & 171 & 0.027 & 31 & 7.4 & 130 \\
\hline & $\mathrm{SiO}_{2}+\mathrm{PMPS}$ & 168 & 0.027 & 31 & 7.9 & 133 \\
\hline & $\mathrm{SiO}_{2}+\mathrm{PMPS}+0.5 \mathrm{ml} \mathrm{DMC}$ & 168 & 0.022 & 16 & 7.3 & 130 \\
\hline & $\mathrm{SiO}_{2}+\mathrm{PMPS}+1.0 \mathrm{ml} \mathrm{DMC}$ & 173 & 0.021 & 14 & 6.6 & 129 \\
\hline \multirow{5}{*}{300} & $\mathrm{SiO}_{2}+\mathrm{PMPS}+1.5 \mathrm{ml} \mathrm{DMC}$ & 191 & 0.024 & 17 & 6.5 & 130 \\
\hline & $\mathrm{SiO}_{2}+\mathrm{PMPS}$ & 190 & 0.028 & 28 & 6.0 & 134 \\
\hline & $\mathrm{SiO}_{2}+\mathrm{PMPS}+0.5 \mathrm{ml} \mathrm{DMC}$ & 160 & 0.023 & 17 & 7.0 & 130 \\
\hline & $\mathrm{SiO}_{2}+\mathrm{PMPS}+1.0 \mathrm{ml} \mathrm{DMC}$ & 170 & 0.022 & 15 & 6.7 & 130 \\
\hline & $\mathrm{SiO}_{2}+\mathrm{PMPS}+1.5 \mathrm{ml} \mathrm{DMC}$ & 178 & 0.022 & 16 & 7.0 & 130 \\
\hline
\end{tabular}

(Figure 2). The content of adsorbed water in the samples synthesized in the presence of alkyl carbonate is smaller, unlike the silica modified in the absence of alkyl carbonate for which the band intensity with maximum at $3450 \mathrm{~cm}^{-1}$ is much higher.

It should be noted that there is no absorption band $\mathrm{O}-\mathrm{H}$ in the free silanol groups at $3750 \mathrm{~cm}^{-1}$ in the spectra of the modified products, indicating their full participation in the reactions with the mixture of PMPS and DMC.

Figure 3(a) shows the adsorption-desorption isotherms of nitrogen for pristine silica and for silica modified with pure PMPS and its mixtures with DMC. The type of isotherm is common for the all modified silica, namely they are characterized by the fourth type according to the Brunauer's classification (a certain superposition of isotherms of II and IV types of the IUPAC classification without plateau adsorption [25] [26]) and the third type of the hysteresis loop in the range of high values of relative pressures [27]. Within the range of relative pressures $p / p_{0}=0.4-0.9$ one can observe lowering of all adsorption-desorption isotherms of nitrogen for modified silicas 


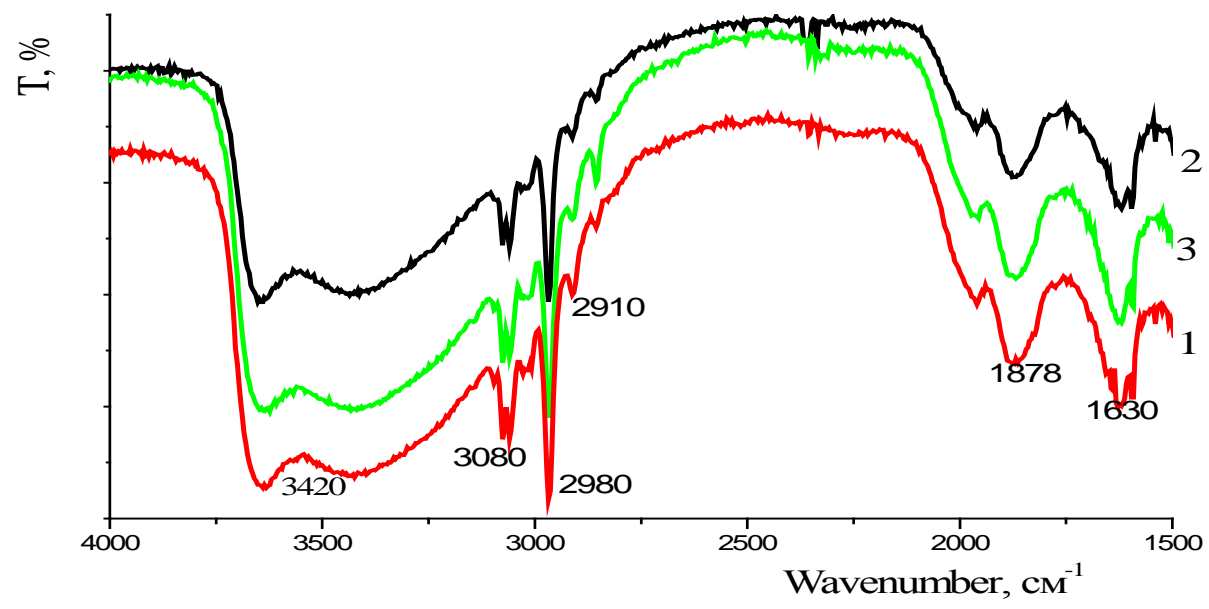

Figure 2. IR spectra of fumed silicas modified with mixtures of PMPS (2 ml) and DMC (0.5 $\mathrm{ml})$ for $2 \mathrm{hrs}$ at $200^{\circ} \mathrm{C}, 250^{\circ} \mathrm{C}$ and $300^{\circ} \mathrm{C}(1-3$, respectively).

in comparison with the pristine silica.

Structural and surface characteristics of the pristine silica and the samples modified with poly(methylphenylsiloxane) are given in Table 1.

The samples synthesized by modification of the silica surface with PMPS have large number of voids with the average radius $R_{\text {ave }}>28 \mathrm{~nm}$. For the samples modified with the mixture PMPS and DMC size-range of voids is $R_{\text {ave }}<17 \mathrm{~nm}$.

Materials obtained by treatment with poly(methylphenylsiloxane) and mixtures of PMPS and DMC with different ratios of the components have similar contact angles of wettings (from $129^{\circ}$ to $134^{\circ}$ ). It indicates the high hydrophobic properties of surface of the synthesized organosilicas (Table 1). As stated before, hydrophobicity of the modified materials is largely associated with the structure of the surface layer and determined by the concentration of the grafted organic groups.

According to the elemental analysis data (Table 1) one can find that using pure poly(methylphenylsiloxane) and mixtures of PMPS with dimethyl carbonate for modification of silica at $250^{\circ} \mathrm{C}$, allows to reach the high concentrations of grafted organic groups in the synthesized samples (the average of carbon content $\sim 7 \mathrm{wt} . \%$ ). It can be explained by the fact that at higher temperature the depolymerisation process becomes more intense, i.e. molecular weight of oligomers is decreased and number of contacts of the modifying reagent with the surface sites is increased. The average of carbon content is smaller ( $\geq 6.6$ carbon) for the samples synthesized at $200^{\circ} \mathrm{C}$ and $300^{\circ} \mathrm{C}$. It can be assumed that at $200^{\circ} \mathrm{C}$ the depolymerisation degree is low what promotes chemisorption of the oligomers with relatively higher molecular weight and with weaker contacts with the surface sites. At $300^{\circ} \mathrm{C}$ an increased number of low-weight oligomers with lower carbon content is formed that affect the value of chemisorption and the structure of the grafted surface layer.

Extreme character of dependence of the carbon content on the modification process temperature is a result both the chemisorption kinetics and the depth of thermal depolymerisation. During modification with the mixture of PMPS and DMC two processes are competing: a thermal and chemical (with participation of DMC) depolymerisation. The degree of modification depends on kinetic features of chemisorption and the process of splitting of polysiloxane chains. At $200^{\circ} \mathrm{C}$ the rate-limiting step of modification is splitting the siloxane bonds in the reaction with dimethyl carbonate with following attachment of oligomers to the silica surface. However, at $250^{\circ} \mathrm{C}$ and $300^{\circ} \mathrm{C}$ the thermal depolymerisation prevails, the quantity of initiator slightly affects the surface carbon content that controls the degree of the siloxane depolymerisation. Generally, with increasing amounts of initiator the degree of the polysiloxane grafting increases, however the temperature $250^{\circ} \mathrm{C}$ is optimal for maximum anchoring of the functional groups to the silica surface.

In Figure 4, the TEM images of fumed silica modified with PMPS and its mixture with DMC are presented. The data of the transmission electron microscopy showed that particles of silica modified with PMPS formed the aggregates with size of 20 - $40 \mathrm{~nm}$ (Figure 4(a)). The structure of fumed silica modified with the mixture of DMC and PMPS consists mostly of smaller particles of 10 - $12 \mathrm{~nm}$ (Figure 4(b)). 

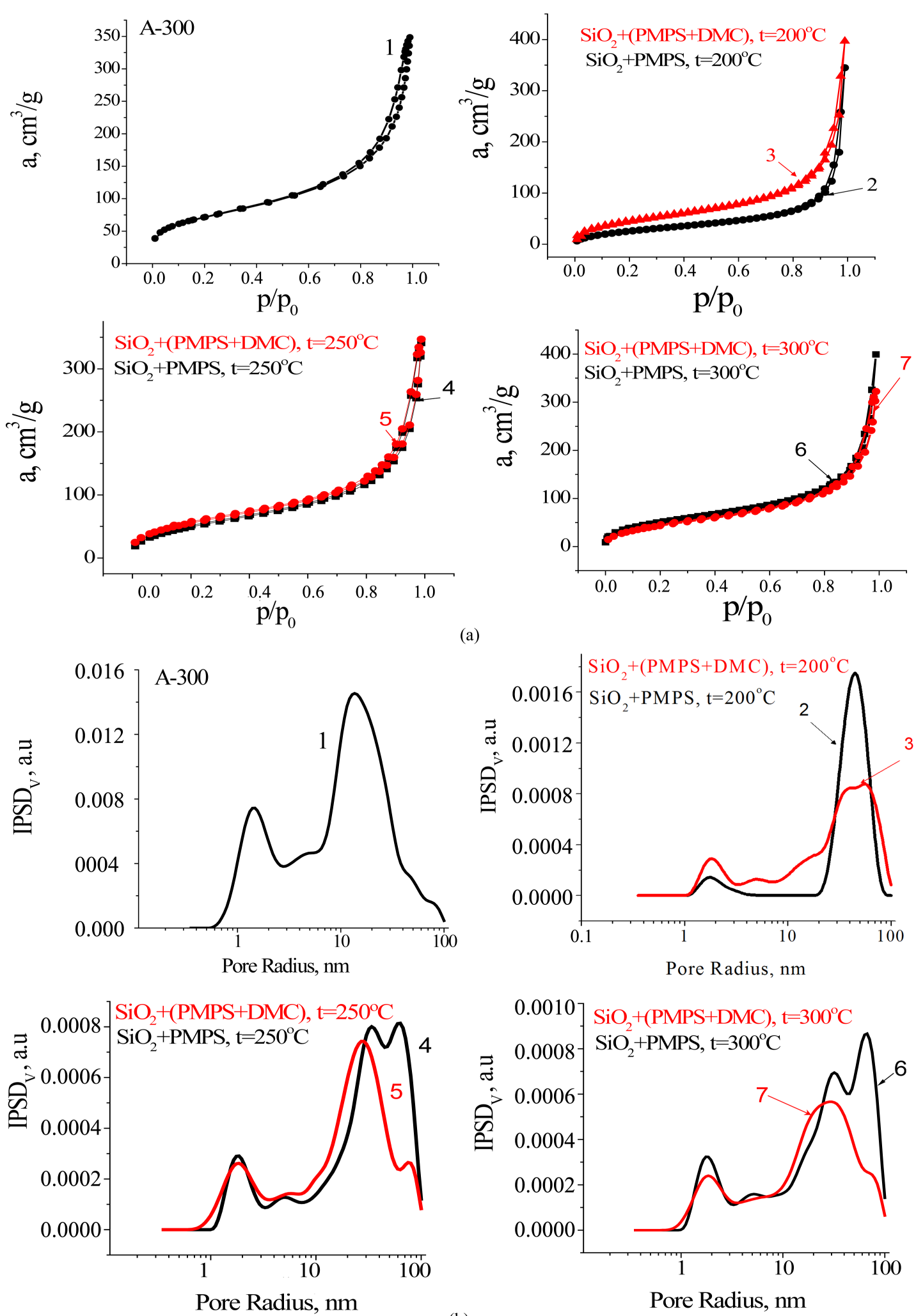

(b)

Figure 3. Adsorption-desorption isotherms of nitrogen at $77 \mathrm{~K}$ (a) and pore size distribution (b) for the pristine silica A-300 (1) and fumed silicas modified with pure PMPS $(2,4,6)$ and with mixture of PMPS and DMC $(3,5,7)$ for 2 hrs at $200^{\circ} \mathrm{C}(2$, 3), $250^{\circ} \mathrm{C}(4,5)$ and $300^{\circ} \mathrm{C}(6,7)$. 
It is known that the atomic force microscopy is crucial in measuring the characteristics of surface topography. In this paper, the microstructure of the surface layer of modified silica was studied by applying the AFM technique. The AFM images obtained for the pristine silica and after its modification with PMPS and PMPS/DMC mixture are presented in Figure 5. On the AFM images it can be observed that for the initial fumed silica (Figure 5(a))

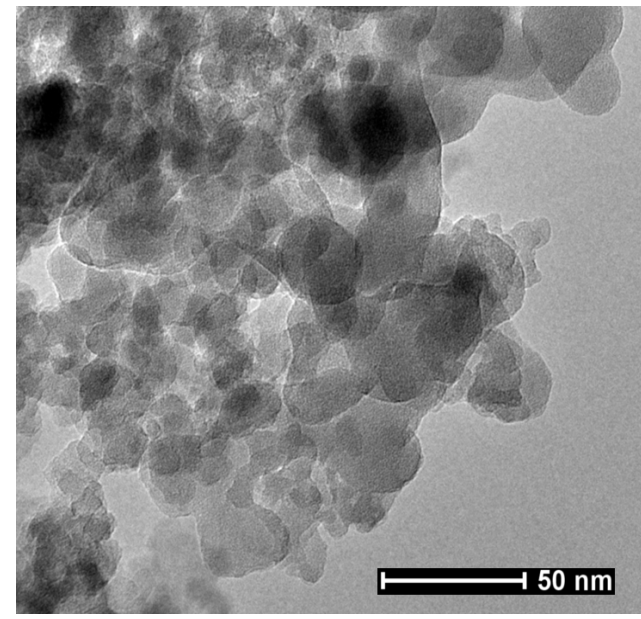

(a)

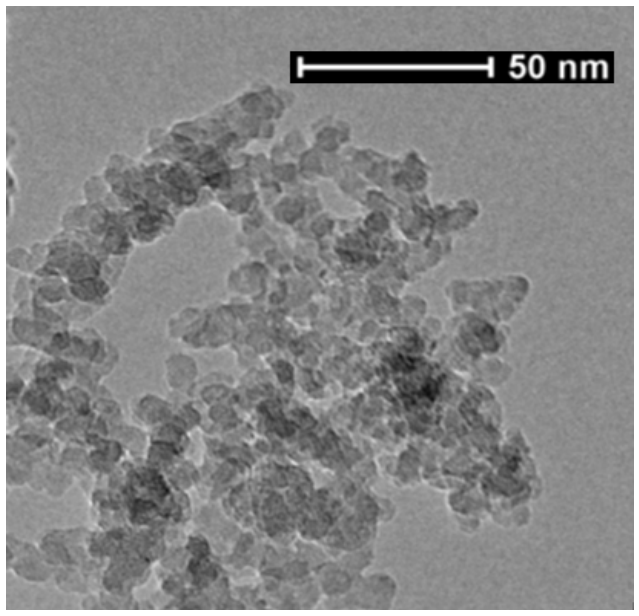

(b)

Figure 4. TEM images of the fumed silicas modified with PMPS (a) or with PMPS/DMC mixture (b) at $250^{\circ} \mathrm{C}$ for $2 \mathrm{hrs}$.

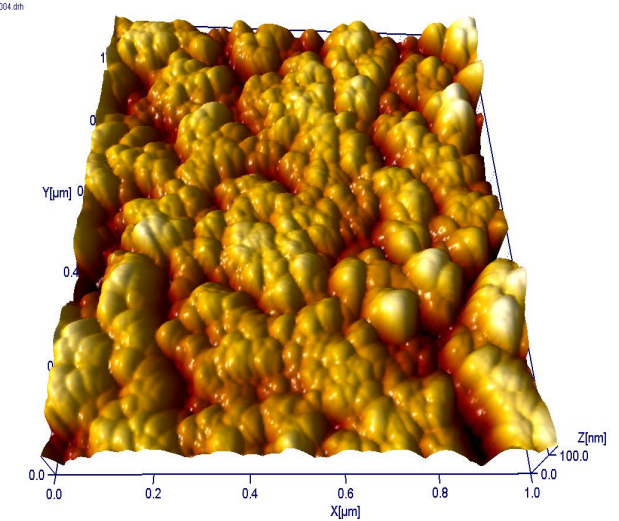

(a)

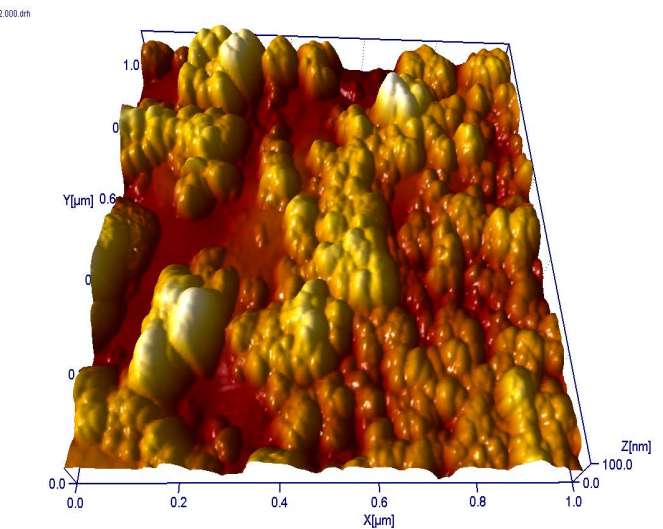

(b)

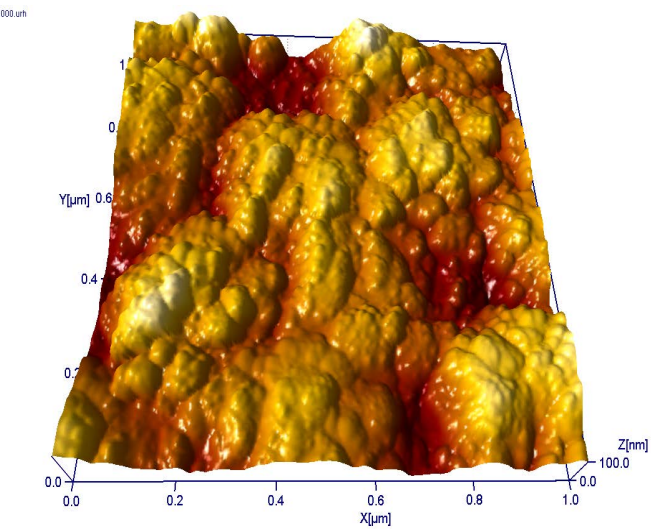

(c)

Figure 5. AFM images of pristine silica A-300 (a) after treatment at $250^{\circ} \mathrm{C}$ for 2 hrs with pure PMPS (b) and with PMPS/ DMC mixture (c). 
the structure of the surface layer is relatively homogeneous. The silica modified with the mixture of DMC/ PMPS is characterized by a narrow size distribution and smallest grain size (Figure 5(c)). The coating microstructure for this sample is rather smooth in comparison to other coatings. After the modification of silica with pure PMPS a wider size distribution (Figure 5(b)) is observed. The particles show tendency to form bigger agglomerates than $\mathrm{SiO}_{2}$ modified with the mixture of DMC/PMPS.

\section{Conclusions}

The properties of modified fumed silicas were studied using the methods of IR spectroscopy, elemental analysis, transmission electron microscopy, nitrogen adsorption-desorption data, atomic force microscopy and measurements of contact angles of wetting. According to IR spectroscopy data, mixtures of PMPS with DMC were shown to provide an increase of the concentration of grafted organic groups in the surface layer and full participation of the free silanol groups in the chemisorptions process at the moderate temperatures $\left(200^{\circ} \mathrm{C}\right)$. At $250^{\circ} \mathrm{C}$, the highest concentrations of grafted organic groups in the synthesized samples (the average of carbon content $\sim 7 \mathrm{wt} . \%$ ) were reached. The average of carbon content is smaller ( $\geq 6.6 \mathrm{wt} . \%$ carbon) for the samples synthesized at 200 and $300^{\circ} \mathrm{C}$. These data indicate that the temperature $250^{\circ} \mathrm{C}$ is optimal for maximum attachment of the functional groups on the silica surface. The quantity of initiator slightly influenced the surface carbon content in the surface modifying layer.

The type of nitrogen adsorption isotherm is common for all modified silica, namely they are characterized by the fourth type according to the Brunauer's classification and the third type of the hysteresis loop in the range of high values of relative pressures. Coating microstructure and morpholology of the modified samples is relatively homogeneous under modification of the silica surface with the mixture of PMPS and DMC. Their structure consists of particles of size $10-12 \mathrm{~nm}$. After the modification of silica with pure PMPS a wider size distribution is observed. Particles of silica modified with PMPS form the aggregates of the size 20 - $40 \mathrm{~nm}$.

\section{Acknowledgements}

The research leading to these results has received funding from the People Programme (Marie Curie Actions) of the European Union's Seventh Framework Programme FP7/2007-2013/under REA grant agreement No PIRSES-GA-2013-612484.

\section{References}

[1] Zenkevich, I.G., Makarov, A.A. and Ivanova, K.V. (2014) Characteristic Variations of Gas-Chromatographic Retention Indices for Phases of Variable Composition. Analytical Chemistry, 69, 1089-1095. http://dx.doi.org/10.1134/S1061934814110148

[2] Yang, M.H., Chen, I.L. and Wu, D.H. (1996) Chemically Bonded Phenylsilicone Stationary Phases for the Liquid-Chromatographic Separation of Polycyclic Aromatic-Hydrocarbons and Cyclosiloxanes. Journal of Chromatography A, 722, 97-105. http://dx.doi.org/10.1016/0021-9673(95)00577-3

[3] Lisichkin, G.V. (1986) Modified Silicas in Sorption, Catalysis and Chromatography. Khimiya, Moscow. (In Russian)

[4] Protsak, I.S., Kozakevich, R.B., Bolbukh, Yu.M. and Tertykh, V.A. (2013) Viscosimetric Study of Polydimethylsiloxane Depolymerization with Dimethyl Carbonate. Chemical Industry, 117, 58-62. (In Ukrainian)

[5] Demianenko, E.M., Grebenyuk, A.G., Lobanov, V.V., Tertykh, V.A., Protsak, I.S., Bolbukh, Yu.M. and Kozakevych, R.B. (2014) Quantum Chemical Study on Interaction of Dimethyl Carbonate with Polydimethylsiloxane. Chemistry, Physics and Technology of Surface, 5, 473-479.

[6] Protsak, I.S., Tertykh, V.A., Goncharuk, O.V., Bolbukh, Yu.M. and Kozakevich, R.B. (2014) Hydrophobization of the Fumed Silica Surface with Polydimethylsiloxanes in the Presence of Alkyl Carbonates. Chemistry, Physics and Technology of Surface, 5, 226-235. (In Ukrainian)

[7] Okamoto, M., Suzuki, S. and Suzuki, E. (2004) Polysiloxane Depolymerization with Dimethyl Carbonate Using Alkali Metal Halide Catalysts. Applied Catalysis A: General, 261, 239-245. http://dx.doi.org/10.1016/j.apcata.2003.11.005

[8] Okamoto, M., Miyazaki, K., Kado, A. and Suzuki, E. (2001) Deoligomerization of Siloxanes with Dimethyl Carbonate over Solid-Base Catalysts. Chemical Communication, 18, 1838-1839. http://dx.doi.org/10.1039/b104371b

[9] Tundo, P., Arico, F., Rosamilia, A.E., Grego, S. and Rossi, L. (2008) Dimethyl Carbonate: Green Solvent and Ambident Reagent. Green Chemical Reactions, 213-232. 
[10] Arico, F. and Tundo, P. (2010) Dimethyl Carbonate: A Modern Green Reagent and Solvent. Russian Chemical Reviews, 79, 479-489. http://dx.doi.org/10.1070/RC2010v079n06ABEH004113

[11] Brunauer, S., Emmett, P.H. and Teller, E. (1938) Adsorption of Gases in Multimolecular Layers. Journal of the American Chemical Society, 60, 309-319. http://dx.doi.org/10.1021/ja01269a023

[12] Nguyen, C. and Do, D.D. (1999) A New Method for the Characterization of Porous Materials, Langmuir, 15, 36083615.

[13] Gun’ko, V.M. (2014) Composite Materials: Textural Characteristics. Applied Surface Science, 307, 444-454. http://dx.doi.org/10.1016/j.apsusc.2014.04.055

[14] Gun'ko, V.M. (2000) Consideration of the Multicomponent Nature of Adsorbents during Analysis of Their Structural and Energy Parameters. Theoretical and Experimental Chemistry, 36, 319-324. http://dx.doi.org/10.1023/A:1005264427135

[15] Gun'ko, V.M., Meikle, S.T., Kozynchenko, O.P., Tennison, S.R., Ehrburger-Dolle, F., Morfin, I. and Mikhalovsky, S.V. (2011) Comparative Characterization of Carbon Adsorbents and Polymer Precursors by Small-Angle X-Ray Scattering and Nitrogen Adsorption Methods. The Journal of Physical Chemistry A, 115, 10727-10735. http://dx.doi.org/10.1021/jp201835r

[16] Do, D.D., Nguyen, C. and Do, H.D. (2001) Characterization of Micromesoporous Carbon Media. Colloids and Surfaces A: Physicochemical and Engineering, 187, 51-71. http://dx.doi.org/10.1016/S0927-7757(01)00621-5

[17] Gun'ko, V.M. and Mikhalovsky, S.V. (2004) Evaluation of Slitlike Porosity of Carbon Adsorbents. Carbon, 42, 843849. http://dx.doi.org/10.1016/j.carbon.2004.01.059

[18] Toth, A., Voitko, K.V., Bakalinska, O., Prykhod’ko, G.P., Bertóti, I., Martinez-Alonso, A., Tascon, J.M.D., Gun’ko, V.M. and Laszloa, K. (2012) Morphology and Adsorption Properties of Chemically Modified MWCNT Probed by Nitrogen, $n$-Propane and Water Vapor. Carbon, 50, 577-585. http://dx.doi.org/10.1016/j.carbon.2011.09.016

[19] Myronyuk, L.I., Myronyuk, I.F., Chelyadyn, V.L., Sachko, V.M., Nazarkovsky, M.A., Leboda, R., Skubiszewska-Zięba, J. and Gun'ko, V.M. (2013) Structural and Morphological Features of Crystalline Nanotitania Synthesized in Different Aqueous Media. Chemical Physics Letters, 583, 103-108. http://dx.doi.org/10.1016/j.cplett.2013.07.068

[20] Gun’ko, V.M., Voronin, E.F., Nosach, L.V., Turov, V.V., Wang, Z., Vasilenko, A.P., Leboda, R., Skubiszewska-Zięba, J., Janusz, W. and Mikhalovsky, S.V. (2011) Structural, Textural and Adsorption Characteristics of Nano-Silica Mechanochemically Activated in Different Media. Journal of Colloid and Interface Science, 355, 300-311. http://dx.doi.org/10.1016/j.jcis.2010.12.008

[21] Sternik, D., Majdan, M., Deryło-Marczewska, A., Żukociński, G., Gładysz-Płaska, A., Gun’ko, V.M. and Mikhalovsky, S.V. (2011) Influence of Basic Red 1 Dye Adsorption on Thermal Stability of Na-Clinoptilolite and Na-Bentonite. Journal of Thermal Analysis and Calorimetry, 103, 607-615. http://dx.doi.org/10.1007/s10973-010-1014-3

[22] Davydov, V.Y. (2000) Adsorption on Silica Surface. In: Papirer, E., Ed., Surfactant Science Series, Marcel Dekker, New York, 63-118.

[23] Gun'ko, V.M., Borysenko, M.V., Pissis, P., Spanoudaki, A., Shinyashiki, N., Sulim, I.Y., Kulik, T.V. and Palyanytsya, B.B. (2007) Polydimethylsiloxane at the Interfaces of Fumed Silica and Zirconia/Fumed Silica. Applied Surface Science, 253, 7143-7156. http://dx.doi.org/10.1016/j.apsusc.2007.02.185

[24] Gao, D., Jia, M. and Luo, Y. (2013) Crosslinked Organosiloxane Hybrid Materials Prepared by Condensation of Silanol and Modified Silica: Synthesis and Characterization. Chinese Journal of Polymer Science, 31, 974-983. http://dx.doi.org/10.1007/s10118-013-1289-5

[25] Condon, J.B. (2006) Surface, Area and Porosity Determinations by Physisorption, Measurements and Theory. Elsevier, Amsterdam.

[26] Sing, K.S.W. (1982) Reporting Physisorption Data for Gas/Solid Systems with Special Reference to the Determination of Surface Area and Porosity. Pure and Applied Chemistry, 54, 2201-2218. http://dx.doi.org/10.1351/pac198254112201

[27] Gregg, S.J. and Sing, K.S.W. (1991) Adsorption, Surface Area and Porosity. 2nd Edition, Academic Press, London. 